\title{
General Psychiatry Barriers and facilitators of peer support work in a large psychiatric hospital: a thematic analysis
}

To cite: Kuek JHL, Chua HC, Poremski D. Barriers and facilitators of peer support work in a large psychiatric hospital: a thematic analysis. General Psychiatry 2021;34:e100521. doi:10.1136/ gpsych-2021-100521

Received 07 March 2021 Accepted 19 May 2021

\section{Check for updates}

(c) Author(s) (or their employer(s)) 2021. Re-use permitted under CC BY-NC. No commercial re-use. See rights and permissions. Published by BMJ.

Health Intelligence Unit, Institute of Mental Health, Singapore

Correspondence to Dr Daniel Poremski; daniel.poremski@mail.mcgill.ca

Jonathan Han Loong Kuek, Hong Choon Chua, Daniel Poremski

\section{ABSTRACT}

Background Peer support workers are an important addition to the mental healthcare profession. However, much of the literature and knowledge of the peer support role is derived from western countries. This concept is relatively new in Asian countries.

Aims The study sought to improve the understanding of the barriers and facilitators of peer support work in a large psychiatric hospital in Singapore.

Methods This study used qualitative data from a larger mixed-methods study. Thematic analysis was conducted based on the five steps recommended by Braun and Clarke (2006): (1) familiarisation of data whereby transcripts were read and reread, (2) generating initial codes, (3) searching for themes by gathering relevant codes, (4) reviewing themes and (5) defining and naming themes.

Results Four subthemes under the broader notion of facilitators (supportive figures, defined role, opportunities for personal growth and identifying personalised coping strategies) and three subthemes under the concept of barriers (unclear role, hostility from non-peer-supportspecialist staff and unsupportive working environments) were identified.

Conclusion Our findings echoed those of previous studies conducted in western countries providing some evidences for the cross-cultural nature of these barriers and facilitators. However, the way their impact can be mitigated or enhanced is likely to be different owing to cultural barriers, such as the general consensus and acceptance of larger personal recovery ideologies. Further research is required in community settings to better understand the boundaries and limitations of our findings. This information will allow us to continue improving peer support worker integration in diverse mental healthcare settings.

\section{INTRODUCTION}

Western mental healthcare systems have increasingly acknowledged and adopted recovery-oriented care models. In 1999, the era's zeitgeist was perfectly captured in The Surgeon General's landmark report on the importance of redefining and re-envisioning mental healthcare. ${ }^{1}$ New care models differentiated themselves from their predecessors in multiple ways. One significant difference was the greater inclusion of people with lived experience of mental illness in service development and provision. The manifestation of such a movement was the utilisation of people with lived experience as formal service providers (ie, peer support workers). This new type of service provider leveraged their unique experiences to support others who may be experiencing similar situations. ${ }^{2-4}$

These newly minted mental health professionals were eventually integrated into a number of services in various mental healthcare settings, such as to support people with mental health conditions ${ }^{5}$, severe mental illnesses ${ }^{46}$ and substance use issues. ${ }^{7}$ Systematic reviews ${ }^{8-12}$ supported these individual findings and demonstrated the effectiveness of integrating peer support workers into larger care teams. As a result of the benefit they bring to multidisciplinary care models, they have been integrated into community mental health teams ${ }^{13} 14$ and supported employment teams ${ }^{15}$, where their presence is counted towards increased programme fidelity.

However, the enactment of these novel peer support roles was accompanied by barriers. These barriers emerged as the discipline evolved and became the focus of scientific enquiry. A review of recovery-oriented practices in hospital settings identified resistance in mental healthcare systems, which maintained a biomedical approach to care, fostered negative attitudes to the concept of recovery and excluded consumers during the development of services. ${ }^{16}$ The institutionalisation of the peer support role and resulting dilution of essential peer support tasks have led to a lack of role clarity in some cases ${ }^{12}$, and by role restriction in other cases their activities must conform with existing care mandates. ${ }^{17} 18$ Other barriers include the lack of professional development and career advancement opportunities, which hinder sustainability and development. ${ }^{19} 20$

It is noteworthy that much of the peer support research originated in western 
countries as the recovery movement stemmed from them, except for Hong Kong, which adopted peer support roles in the early 2010s. ${ }^{21}{ }^{22}$ Even China and India, the two largest countries in Asia, have only recently begun to explore the feasibility of peer support work within their respective cultural contexts. ${ }^{6}{ }^{14}$ This lack of development raises concerns about whether existing literature and knowledge are sufficient to inform the successful implementation of peer support services in Asian contexts.

Certain cultural differences are likely to influence the way in which peer services are implemented and adopted. Notably, because of the more substantial social stigma and lack of literacy surrounding mental health concerns that characterise some Asian settings, the idea of including a former service user in formal mental healthcare processes may be resisted. Furthermore, a scoping review on personal recovery experiences in Asia highlighted the lack of recovery-oriented literature in the Asian context and the need for more culturally grounded lenses to be applied when examining the concept of personal recovery in such a diverse region. ${ }^{23}$ Hence, this study's goal was to identify barriers and facilitators to a peer support role in Singapore's only tertiary psychiatric hospital, thereby filling a gap in the literature relating to peer support work in Singapore and South East Asia.

\section{METHODS}

We used qualitative data collected as part of an overarching quasi-experimental mixed-methods study designed to explore the impact and implementation of peer support work within the Institute of Mental Health (IMH). The larger study assessed the impact of peer support work on the mental health of service users, the attitudes of service providers and the work-life experiences of peer support specialists (PSSs). This study used data obtained as part of that latter component to fulfil its objectives. To guide this study's reporting, we used the Consolidated Criteria for Reporting Qualitative Research checklist. ${ }^{24}$ Before the study began, ethics approval was obtained. Participants gave written informed consent during the first interview; verbal consent was sought and given before each subsequent interview to ensure participants were not feeling coerced to follow up.

\section{Setting}

This study is hosted within IMH where, prior to PSS certification, certain departments such as the addiction clinic, first-episode psychosis ward and mood disorder unit, had already begun using PSSs as part of their care processes, most notably in mutual support groups. In 2016, a tripartite alliance of institutes in Singapore consisting of the National Council for Social Services, the Singapore Association of Mental Health and IMH introduced a formal PSS training and certification course. Although the idea of peer support work predated this initiative, this course was the first in Singapore to develop and aim for the sustained incorporation of certified PSSs in the country's mental healthcare network. People who received this training experienced some form of mental health challenge but tended to be further along their recovery journey. The course covered fundamentals of service provision, as well as peer support-specific roles, such as crafting narratives, managing their dual role as service user and provider and managing the pressures of working in a team.

In 2017, IMH began investing greater efforts to better integrate PSSs into several departments such as psychosocial rehabilitation, emergency services, case management, community-based services, occupational therapy and inpatient/outpatient clinics. The diverse programmes into which they were implemented led to a diversity of roles, but each PSS retained at their professional core that is the idea of using lived experience to support those who were currently experiencing mental illness. They all have direct roles to support service users and only engage in administrative duties insofar as required to document their activities (completing case notes). To maximise their impact, PSSs were placed in departments or programmes similar but not identical to their personal service use experience. Consequently their experiences resemble those of their service users. Currently, IMH has the largest single employment pool of PSSs with 18 members as of December 2020.

\section{Sampling}

To be eligible for our study, people had to be working in a peer support role at our institute. By extension, they met the institute's criteria for employment as a PSS. These criteria included completion of PSS certification, as per course mentioned above, and a period of stability (defined as the absence of relapse which required hospitalisation) lasting a year or more. Additionally, they were formally employed by IMH as salaried employees and worked under the hospital's formal treatment framework.

People were ineligible if they had yet to complete their 3-month probationary period or if they were hired at the hospital before 2017. This decision was taken because prior to 2017, the organisational culture differed and PSSs were ad hoc employees.

We interviewed a final sample of 10 PSSs, as 7 did not meet the participation requirements and 1 declined participation. The enrolment flowchart is presented in figure 1 .

\section{Interview process}

Participants participated in three individual interviews between April 2019 and April 2020, separated by 4-month gaps (baseline, 4 months and 8 months). The interviews lasted a mean (SD) of 63 (20) min. Baseline interviews focused on getting to know the PSSs and their recovery journey. We also inquired extensively about how and why they became a formal PSS. The second and third interviews focused on their service provision experiences within the hospital. We relied on a theoretical framework based on PSS service fidelity from a previous study ${ }^{25}$ to guide the interview. This framework included all the 
18 peer support specialists within the organization:

1) have a history of mental illness

2) have been certified under a local PSS program

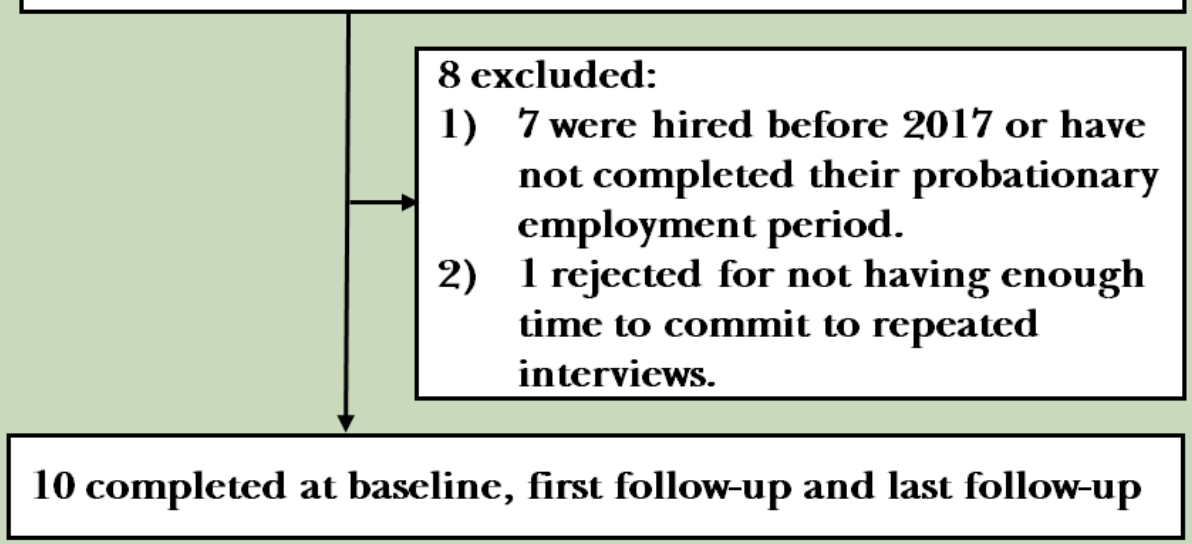

Figure 1 Recruitment flow chart. PSS, peer support specialist.

elements essential to high-fidelity PSS implementation allowing the interview to cover the full range of essential activities. The same interviewer, the senior author, conducted all interviews one-on-one, which accelerated the rapport building across time points and provided more safety for participants to openly share their views. Following each interview, the interviewer generated field notes and reflections to capture key thoughts and ideas that required developing in subsequent interviews.

\section{Reflexivity}

We acknowledge and recognise the possibility of confirmation bias, which may lead the team to focus on favourable results, supporting the PSS programme's continued implementation in its present iteration. We are also aware of the tendency to attend more to positive stories, which might mask valuable information about potential improvement. To address this issue, we were sensitive to stories that began with 'everything is fine' and made specific efforts to probe deeper into the challenges people faced. We specifically devoted additional time to drawing out content from participants who were not immediately forthcoming with the challenges they encountered. However, we were also aware of the potential to stray into interrogatory modes of questioning and sought a balance between extracting information and respecting the information participants wished to volunteer. It should also be noted that none of the researchers involved had vested interests in the success or failure of the movement in the hospital, even if we all had an interest in the recovery movement in "general, and neither was the interviewer in a managerial position with the organisation. Hence, we are confident that the participants were truthful and transparent, and we have not identified any potential reasons for PSSs to skew or hide any responses. For transparency, two other manuscripts use the same dataset. ${ }^{1826}$

\section{Data analysis}

We transcribed and coded the interviews using NVivo V.11. ${ }^{27}$ Grammatical corrections were made to the quotes presented below, which were initially transcribed verbatim. Interview transcripts were coded independently by the first and senior author. We took direction from Braun and Clarke's ${ }^{28}$ recommendations for conducting a thematic analysis while conducting our analyses. This process comprised five phases: (1) familiarisation of data whereby transcripts were read and reread, (2) generating initial codes, (3) searching for themes by gathering relevant codes, (4) reviewing themes and (5) defining and naming themes. Throughout the analysis, we coded the transcripts around two macro-categories, namely boundaries and facilitators, and subthemes were formed based on relevant codes. Constant discussion occurred after the initial inductive coding phases as the two coders/authors continuously refined the respective subthemes. This allowed interviews to be adjusted to query the soundness of the themes we were developing. We relied on principles of primacy and frequency to assign importance to codes. For example, barriers that surfaced immediately when people were asked about their challenges (primacy) were given more weight than those that emerged further along in the discussion. Barriers that appeared more frequently were also given more weight than those mentioned infrequently. We balanced these two principles to arrive at a list of barriers and facilitators that were supported by evidence and represented the greatest number of participant experiences.

\section{RESULTS}

We interviewed 10 PSSs (six women, four men) who had a mean (SD) age of 30 (6.2) years experienced various 
diagnoses such as schizophrenia, depression, bipolar disorders, addiction disorders and obsessive-compulsive disorders. The ethnicity of the participants reflected that of the Singapore population, including Chinese, Malay and Indian. All scheduled interviews were completed, and none of the PSSs were admitted during the follow-up period or within their year preceding employment as a PSS in the hospital. One participant left his job, and his last interview session was not audio recorded according to his wishes.

We identified four subthemes under facilitators (supportive figures, defined role, opportunities for personal growth and identifying personalised coping strategies) and three subthemes under barriers (unclear role, hostility from non-PSS staff and unsupportive working environments).

\section{Facilitators}

\section{Supportive figures}

Non-peer staff, in particular, supportive team supervisors and working partners were vital to ensuring that PSSs felt supported during challenging times at work and provided them with greater autonomy over what they were allowed to do. Furthermore, having their support ensured that PSSs could secure various resources, such as locations to conduct their support sessions or materials for use in the sessions.

I think my partner did a great job to support my intro to the team. It's like she did a lot of chasing for me, like getting my id tag done, and my access. Yeah, so it's like she's kind of my guardian for that first maybe one or two months. So I was quite well adjusted like to the team and all that. She takes care of me very well and I think that really helps when there is a pair, when we have a pair in the unit. (50001)

There were days where I really didn't want to get to work because of that, and no matter how hard I pushed myself. So having a supervisor and reporting officer who's very understanding of that was I think, monumental in me carrying on with what I'm doing. (50002_2)

So when I ask for help, I mean the ward manager was very helpful and helped us do a lot of stuff. For example, a cabinet key, I just need somewhere to put my stuff for example. So we just keep asking. Because I don't know who to ask so we keep asking maybe the wrong people, in the end I found the ward manager and she gave us a cabinet key which solved a lot of problem. (50006)

They also doubled up as mediators to ease tensions between PSSs and non-peer staff by explaining both parties' perspectives and allowing them to find common ground and a shared understanding of how to function as an effective team.

I think lots of supervision, good supervision from a supervisor helps, to see things from their perspective, from a clinician's perspective, from a doctor's perspective, a CM's perspective, and taking that in to account but yet at the same time, not discounting the effort they have done. (50004)

....is also understanding what are the different... what the other roles are looking for as well, what is the case manager looking for, what will be important to a case manager, what will be important to a doctor, what will be important to like a OT. So after knowing that, I kind of know how to frame the information, thinking about it like "oh yeah, this might be helpful" so I kind of pen it down, maybe write some suggestions. (50005)

\section{Defined role}

Gaining clarity and a better understanding of their role provided PSSs more confidence in carrying them out successfully. It also allowed for the formation of muchneeded boundaries and helped them coexist harmoniously with non-peer staff, improving professional relationships between all parties involved.

I think even before we start the work or anything, that has to be established, if like "what role am I playing, I'm a peer support specialist, I'm not a patient anymore". That has to be talked about, even before a person starts his work. I think that would help really make the role very clear that whatever session ... I think it's just really the boundaries, really draw the lines very clearly, where we're coming in from. (50005)

I make it quite clear, when I have my follow-up consultation, I try not to talk about work, but he's the one who keep asking me about work. So he'll ask me how's your work at the department. So I'll say okay, I'll tell him. But there's a difference between what I told him in a consultation room vs what I told him as a staff. (50006)

At times, this involved creating a role, when none existed, within existing multidisciplinary teams and determining the boundaries, scope of service and administrative procedures. This process was done in partnership with other mental health professionals.

So we have let's say for a support group, for us we have created procedures and processes on how to go about running the group, what is the intention of the group, if safety is something that is important for the group, how do we ensure safety. If issues are being raised up in the group among the peers, how do we address it, all that. so we look into processes, and we evaluate, you know, is the process and procedure effective in what we want to accomplish. (50004)

I kind of feel that if we make ourselves productive, and not just sit back and wait till people ask you if you can do things, I don't think we need to wait till then, there are a lot of things to do, at least on my side, because there are a lot of duties other than PSS 
bound work, even administrative duties which need to be done, and since a PSS is a support role, I guess we can support any other discipline or any other type of work in whatever way that we can. (50007_3)

\section{Opportunities for personal growth and finding meaning}

All our participants shared a desire to receive more training and professional development opportunities specific to their PSS role. However, there were existing courses, conferences and workshops that they could attend within their respective departments.

So other skills, for me I think specifically it would be good for me to....something that i'm really interested in is micro counselling skills, yeah... that's something that has been... we've been talking about because that would be good. Another one would be motivational speaking skills to add on. The third one would be group facilitation, because as group numbers get bigger it gets more challenging, and I run groups alone, I don't have another person to run groups with me. (50002)

Interviewer: But do you go for the courses that they have here? IMH, sort of the generic courses they might have? Participant: Generic will be like the heartsaver and all that. I'm going for motivational interviewing next week, so I'm not sure how. It's different because I think if it's a course that's specific for peer support specialist it's very catered for us, the way how we do, the peer support element and all that, but if it's like the generic one is... it's not that they're not good, but I will hope they will have something for the peer support. (50001_2)

They also voiced the need for meaningful work and feelings of personal growth to sustain their motivation as PSSs. These moments of change occurred mostly through introspection or periods of reflection when they stopped and took stock of their journey as PSSs thus far.

My supervisor wholeheartedly has a trust that I'm capable of doing my job, which is something I feel is... you know, very important to not only help a peer support specialist grow, but it also gives you the avenue then to explore other possibilities. (50002)

But then it is also a process of sowing seeds, it's also inviting the idea of recovery and also like... and all that is meaningful to me and rewarding, because I get to do that... I felt that many other jobs didn't give the same meaning and purpose, the way this job has. (50004)

\section{Identifying personalised coping strategies}

Lastly, finding suitable operating methods that worked for them within the circumstances they were placed into, and identifying relevant coping techniques to buffer from stressors in their lives, would help them sustain their well-being and allowed them to continue providing PSS services.
So after I got back to work, something that I felt was very essential to do was to revisit something called a wellness action plan which was done in the beginning with my clinical supervisor, and that was basically to identify when I'm doing well and when I'm not doing well at work. (50002_2)

I think for myself, I think I just had people to go to, to process some of these emotions. So whenever I heard these stories about like hearing these experiences from my peers, I really made it a point to say that if I really felt bad about it or any uncomfortable emotions, I will go process it with someone, so whether it's like colleagues or my friends, just talk about my uncomfortable feelings. (50005)

I would prefer to look at it (the work) as a positive kind of pressure, to make me improve and not get unwell. It sort of like a good kind of pressure for me to better and get better and better and not sort of relapse. (50008)

\section{Barriers}

\section{Unclear role}

Perhaps the most salient barrier, all the PSSs mentioned, in some form or another, was the lack of clear direction and role boundaries regarding the role they were meant to play in their respective teams. They grappled with this uncertainty and for some of them, being the pioneer batch of PSSs meant having to build a foundation of expectations about what PSS work entailed. This confusion was also further complicated when their supervisors were not familiar with the notion of PSS.

But my experience being in the practicum as the first, or one of the few new PSS that is going into an organization or department, it's going to get chaotic. Because half the time, you don't know what you're supposed to be doing exactly, the supervisor doesn't know what he's supposed to be doing exactly, and it seems like there's just two confused people coming together trying to make things work. (50004)

Like what you mentioned about the clarity of the role. I think when we first came in, me and my fellow colleagues, we didn't really know how we fit into the system. So it seems like, even now we're doing a bit of everything. (50005)

Confusion surrounding the boundaries of the PSS role also led to tension between PSSs and their non-peer colleagues. Particularly, overlaps or similarities in responsibilities were often highlighted or viewed as a threat.

We will discuss our challenges that we have with our clients but on top of that we also talk about, grey areas like, sometimes I will ask her "I'm not sure if I'm actually stepping the boundaries of the case managers or social worker", or "am I doing this thing right?" (50001) 
Sometimes staff might feel that we are overshadowing or we're overpowering them, maybe they feel a bit threatened. (50007)

Furthermore, owing to the nature of the PSS role, there were times when PSSs ended up being colleagues with non-peer staff who had previously been part of their mental health team. This circumstance led to dissonance and difficulty in adapting to a new partnership paradigm, and there often were no guidelines on how they should be interacting with each other.

The most challenging part is sometimes getting used to the idea that "oh my family therapist kind of person is now my supervisor". The idea that "oh my case manager last time now sits opposite to me at work". Like the brain needs to take a while to even get used to like what's going on. (50004)

At first it was quite difficult to figure it out, the mixing of roles, like my psychologist treated me and I'm now working with him. But it takes a bit of time to get used to it, to feel that actually I can now help people that once. (50008)

While policies exist to reduce the chance that a PSS works with a former service provider, the fluid nature of staff positions means that despite not working in a ward in which they had been treated, PSSs may encounter staff that have transitioned to other roles.

\section{Hostility from non-PSS staff}

Many of our participants also reported facing some form of perceived hostility, at times bordering on discrimination from non-PSS staff. For example, one PSS shared how a former service provider suggested that she not provide services to others, as she was once a service user herself.

...even to the point where I got a nasty remark by one of the nurses and this was a nurse I knew from previously, for a long time. she said something nasty to me, something like "you shouldn't be here, you shouldn't be like here". I was like, "wow okay I'm just trying to do my job here and I hear a comment like that", and from someone who I actually knew before so it was really hard to deal with. (50002)

Such experiences were not uncommon as other PSSs also shared similar negative feelings when providing input during multidisciplinary team meetings. They described needing to be extra careful not to offend anyone when they offered their opinions.

I think one of the challenges that comes out during that setting is that when we don't deliver in a way that is easy to listen and accept for people, they get too defensive, they take it personally and it make it seems as if "oh, you're trying to tell me to do my job" or "you're telling me I'm not doing my job" you know, but it's not what we intend. (50004)
I still feel it's a bit hard, because most of the people they come from a professional kind of perspective. So it's a bit hard to fit in, really fit in in terms of the discussion because I don't really know how to provide my inputs to them. Because It's just me, one against all the professionals, so sometimes I might feel a bit like soft, more soft-spoken compared to them. (50008)

Beyond the overt forms of hostility, subtler issues included being excluded from office chat groups. Some participants also noticed that non-peer staff were more welcoming of new non-peer staff than PSSs.

So I didn't think that much of that until about 6 months later when I go to the multidisciplinary team then they include me into the whatsapp group, then I realized that every single new staff that comes in, even if they are a new intern, they get into the whatsapp group. Then I realize that “oh, that's what's going on”. (50006)

Sometimes, it's hard for others to sometime accept... how could they work with an addict? Some cannot accept it. Because being an addict is really a big stigma, a big stigma. It's worse, in my own opinion, it could be worse than those who are suffering from mental illnesses, because in many instances, in the Asian context, people find that being a drug addict is not a disease, you choose to be an addict, nobody force you to take the drug. (50003)

\section{Unsupportive working environments}

Although interpersonal relationships did cause some discomfort to PSSs, at times, certain practices or normative behaviours also hindered the practical introduction of peer support-focused mode of mental health support.

I thought that was a little bit sad, because it kind of cuts off the option who may want to join groups but they can only join my group if they are referred through a psychologist. Because there were some people who were interested to join my group but they were not seeking psychotherapy services here. (50002)

Participants also faced challenges while starting programmes or carrying out certain services because of multiple bureaucratic barriers. These challenges gave the participants the impression of mistrust towards PSSs.

...I still need to go through like my supervisor or like department head, the person in charge, to say whether it's okay or not okay, and why is it okay or not okay. So I still have to go through that level. But the western idea of co-production right, is just you and me, we come together, brainstorm, everything together from scratch, execute it and we run with it, that's it, we don't need to seek a second opinion about a non PSS, a high profess... I mean if we do it's great, you have greater insights, but not as a capacity of "I give the green light, no, I don't give the green light, 
orange light, yeah". There's still a bit of restriction and gatekeeping there. (50004)

Their working environment and the institute's siloed structure also hindered their ability to operate across various departments, something that may be essential to continuity of care.

\section{DISCUSSION}

\section{Main findings}

The barriers we identified were quite similar to previous findings on PSS work implemented within hospital settings despite the differences in cultures. For example, a systematic review $^{12}$ revealed that organisational culture and its issues were a significant hindrance when integrating them within care teams. Our study yields similar results as the three barriers which we identified (unclear role, hostility from non-peer staff and unsupportive working environments), were primarily because of the organisations' pre-existing culture (ie, greater emphasis on a biomedical perspective). Addressing these barriers could lead to more changes to existing frameworks and protocols to introduce more recovery-oriented and collaborative strategies.

In Singapore, however, the PSS role remains a novel concept and in its developmental stages. From our study, it was evident that other mental healthcare professionals often did not know or understand much about the role. At times, our participants even felt that healthcare professionals rejected the notion that someone with a mental health condition should provide services to others, echoing social and cultural opinions of people with mental illness held by service providers in Singapore ${ }^{29}$ and in Asia in general. ${ }^{23}$ Hence, these beliefs lead to wideranging disagreements on various aspects such as how the role should evolve, concerns raised by other mental healthcare professionals on the inclusion of PSSs within the system and how effective PSSs are as formal care professionals. ${ }^{8}$ Such debates will continue to hinder the progress towards removing some of the barriers that exist.

Existing attitudes can be challenging to modify and culturally difficult to shift. For example, one PSS mentions how ' ... in the Asian context, people find that being a drug addict is not a disease, you choose to be an addict, nobody force you to take the drug', which caused people around him to still view him negatively as a former substance user despite now being a formal mental health professional. These ingrained belief systems severely impact their credibility and integration within a larger mental health workforce. Nevertheless, it is worth noting that some researches have highlighted how greater exposure to working with peers or including them in more aspects of service provision can improve attitudes. ${ }^{30} 31$ Hence, an intentional effort could be made to include PSSs in more aspects of an institution's onboarding processes, such as allowing them to conduct orientation programmes, traditionally hosted by human resource departments, which may aid newer employees in being aware and gaining knowledge of this new group of mental health professionals.
The facilitators we found (supportive figures, defined roles, opportunities for growth and personalised coping strategies) were also in line with previous studies ${ }^{12} 16$, adding credence to our findings and the generalisability of these factors integral to the well-being and motivation of PSSs. Notably, they need to be supported on an organisational level in providing adequate and relevant training opportunities to improve their capacity as PSSs. For example, developing leadership pathways could further involve PSS in an organisation's various operations. However, certain factors, such as managing a dual relationship and addressing potential stigmatisation owing to their mental health experiences, need to be considered (see the study by Jenkins and colleagues for the full list they identified ${ }^{20}$ ).

A particular finding not commonly observed in other studies was that our participants each had a unique coping method, ranging from talking to their families to relying on other activities such as engaging in their hobbies. This demonstrates congruence with the uniqueness of the notion of recovery within which they are trained as PSSs. ${ }^{1}$ Leveraging these findings, we could turn our attention to training PSS supervisors to adequately engage and support them in identifying personalised coping strategies and techniques to maintain their well-being and motivation to continue providing services as PSSs. This would also further enhance the connectedness and supportive potential observed in another of our facilitator themes, 'supportive figures'.

\section{Limitations}

Our study has limitations. Notably, our sample of PSSs came from the pool of PSSs who were operating within IMH. Community-based PSSs may have very different experiences as the challenges and scope of their roles may vary significantly between agencies.

Another possible limitation concerns the PSS-specific theoretical framework we used to approach the topic. The implementation literature is extensive and treating PSSs as a generic workforce without special consideration for their peculiar dual role might have led us to select a different implementation framework. Such a framework might have returned different barriers that align with the implementation literature. It is possible that an entirely theoretical inductive approach could lead to new results that are culture specific. However, arguing for such a ground-up approach may be difficult given the similarities that exist within the concepts of recovery and the parallel execution of PSS services and medical services in general.

A final limitation concerns generalisability. andBecause of our isolated sample and the fact that IMH is Singapore's only tertiary psychiatric hospital, we are unsure how generalisable our findings may be to other mental health settings in nonpsychiatric hospitals. However, our results largely echo those previously documented in the literature, suggesting that there may be generalisability, even cross-culturally. Future studies can expand on our findings by exploring barriers and facilitators to integrating PSSs in community-based settings where the PSS role and activities may be quite different from a hospital setting. Complementing this study in such a way 
would also allow better recommendations to be put forth to facilitate continuity of care in setting where the PSS role may extend beyond the institutional setting, straddling community and hospital services.

\section{Implications}

Being the first study in Singapore to examine various barriers and facilitators to implementing peer support programmes in a mental health setting, we hope that our findings will generate more conducive dialogue on changes that need to be made to existing policies and operation frameworks. These difficult conversations are especially important in Asian cultures where hierarchy, tradition and culture are deeply interwoven with mental healthcare systems and policies that need to be addressed over the course of PSS integration efforts. For example, knowing the barriers to peer work, measures can be introduced to mitigate their impact. Implementing an introductory course to the recovery movement and peer workers' roles could be a good step to improve PSS working environments. Increasing their legitimacy through certification may not be enough and efforts need to be made to help existing staff acknowledge and embrace PSSs as another type of mental health professionals. Additionally, the facilitators we identified could be enhanced by introducing more relevant courses to PSSs. This would ensure that they can develop professionally and legitimise the PSS role as a formal mental health profession with its relevant development pathways and career trajectories.

\section{CONCLUSION}

Peer support workers are an integral component of recovery-oriented mental health institutions. Our study provides insight into the barriers and facilitators of PSS work within a tertiary psychiatric hospital setting in a multi-ethnic Asian country. Our results would benefit from corroboration from future studies in community settings. This would provide a more holistic picture of factors that impact the work of PSSs across healthcare settings. As the peer support movement continues to grow in Singapore, additional efforts are necessary to ensure we gain a better understanding of how to support PSSs and facilitate their wider sustained integration.

Contributors JHLK analysed the data, developed the specific research questions, interpreted the findings, wrote the first draft of the article and approved the final draft. HCC developed the research project, acted as a mentor for the grant holder, interpreted the findings, edited the first draft and approved the final draft. DP designed the study, collected the data, obtained funding, wrote the second draft and approved the final draft.

Funding This research was funded by the Singapore Ministry of Health, National Medical Research Council Health Service Research New Investigator Grant (HNIG17Nov004).

Competing interests None declared.

Patient consent for publication Not required.

Ethics approval Ethics approval was obtained from the relevant institutional (\#646-2018) and national ethics review committees (\#2018/01131).

Provenance and peer review Not commissioned; externally peer reviewed.
Data availability statement Data are available upon reasonable request.

Open access This is an open access article distributed in accordance with the Creative Commons Attribution Non Commercial (CC BY-NC 4.0) license, which permits others to distribute, remix, adapt, build upon this work non-commercially, and license their derivative works on different terms, provided the original work is properly cited, appropriate credit is given, any changes made indicated, and the use is non-commercial. See: http://creativecommons.org/licenses/by-nc/4.0/.

ORCID iD

Daniel Poremski http://orcid.org/0000-0003-4492-0442

\section{REFERENCES}

1 Davidson L. The recovery movement: implications for mental health care and enabling people to participate fully in life. Health Aff 2016;35:1091-7.

2 Repper J, Carter T. A review of the literature on peer support in mental health services. J Ment Health 2011;20:392-411.

3 Salzer MS, Schwenk E, Brusilovskiy E. Certified peer specialist roles and activities: results from a national survey. Psychiatr Serv 2010;61:520-3.

4 Davidson L, Chinman M, Kloos B, et al. Peer support among individuals with severe mental illness: a review of the evidence. Clinical Psychology: Science and Practice 1999;6:165-87.

5 Barr KR, Townsend ML, Grenyer BFS. Using peer workers with lived experience to support the treatment of borderline personality disorder: a qualitative study of consumer, carer and clinician perspectives. Borderline Personal Disord Emot Dysregul 2020;7:20.

6 Fan Y, Ma N, Ma L, et al. Feasibility of peer support services among people with severe mental illness in China. BMC Psychiatry 2019;19:360.

7 Humphreys K, Wing S, McCarty D, et al. Self-Help organizations for alcohol and drug problems: toward evidence-based practice and policy. J Subst Abuse Treat 2004;26:151-8.

8 White S, Foster R, Marks J, et al. The effectiveness of one-to-one peer support in mental health services: a systematic review and meta-analysis. BMC Psychiatry 2020;20:534.

9 Simmons MB, Grace D, Fava NJ, et al. The experiences of youth mental health peer workers over time: a qualitative study with longitudinal analysis. Community Ment Health J 2020;56:906-14.

10 Shalaby RAH, Agyapong VIO. Peer support in mental health: literature review. JMIR Ment Health 2020;7:e15572.

11 Schlichthorst M, Ozols I, Reifels L, et al. Lived experience peer support programs for suicide prevention: a systematic scoping review. Int J Ment Health Syst 2020;14:65.

12 Ibrahim N, Thompson D, Nixdorf R, et al. A systematic review of influences on implementation of peer support work for adults with mental health problems. Soc Psychiatry Psychiatr Epidemiol 2020;55:285-93.

13 Wright-Berryman JL, McGuire AB, Salyers MP. A review of consumerprovided services on assertive community treatment and intensive case management teams: implications for future research and practice. J Am Psychiatr Nurses Assoc 2011;17:37-44.

14 Fan Y, Ma N, Ma L, et al. A community-based peer support service for persons with severe mental illness in China. BMC Psychiatry 2018;18:170.

15 Kern RS, Zarate R, Glynn SM, et al. A demonstration project involving Peers as providers of evidence-based, supported employment services. Psychiatr Rehabil J 2013;36:99-107.

16 Lorien L, Blunden S, Madsen W. Implementation of recovery-oriented practice in hospital-based mental health services: a systematic review. Int J Ment Health Nurs 2020;29:1035-48.

17 Adams WE. Unintended consequences of institutionalizing peer support work in mental healthcare. Soc Sci Med 2020;262:113249.

18 Poremski D, Kuek JHL, Qi Y, et al. A longitudinal qualitative analysis of the way peer support specialist roles change over time. Administration and Policy in Mental Health. under review.

19 Jones N, Kosyluk K, Gius B, et al. Investigating the mobility of the peer specialist workforce in the United States: findings from a national survey. Psychiatr Rehabil J 2020;43:179-88.

20 Jenkins GT, Shafer MS, Janich N. Critical issues in leadership development for peer support specialists. Community Ment Health $\mathrm{J}$ 2020;56:1085-94.

21 Chang TCS, Liu JSY. Beyond illness and treatment. East Asian Arch Psychiatry 2014;24:125-7.

22 Tse S, Tsoi EWS, Wong S. Training of mental health peer support workers in a non-Western high-income City: preliminary evaluation and experience. International Journal of Social Psychiatry 2014;60:211-8. 
23 Kuek JHL, Raeburn T, Wand T. Asian perspectives on personal recovery in mental health: a scoping review. J Ment Health 2020:1-17.

24 Tong A, Sainsbury P, Craig J. Consolidated criteria for reporting qualitative research (COREQ): a 32-item checklist for interviews and focus groups. Int J Qual Health Care 2007;19:349-57.

25 Chinman M, McCarthy S, Mitchell-Miland C, et al. Early stages of development of a peer specialist fidelity measure. Psychiatr Rehabil J 2016;39:256-65.

26 Poremski D, Kuek J, Qi Y, et al. The impact of peer support work on the mental health of peer support specialists. Journal of Mental Health. under review.
27 QSR International Pty Ltd. NVivo 11 plus, 2017. Available: http:// www.qsrinternational.com/

28 Braun V, Clarke V. Using thematic analysis in psychology. Qual Res Psychol 2006:3:77-101.

29 Yuan Q, Picco L, Chang S, et al. Attitudes to mental illness among mental health professionals in Singapore and comparisons with the general population. PLoS One 2017;12:e0187593.

30 Bunger AC, Navarro El, Lewis CC. How do Peers shape mental health clinicians' attitudes toward new treatments? Adm Policy Ment Health 2021;48:440-9.

31 Klee A, Chinman M, Kearney L. Peer specialist services: new frontiers and new roles. Psychol Serv 2019;16:353-9.

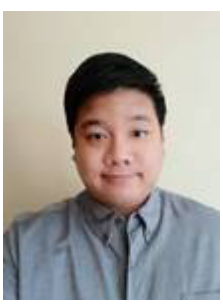

Jonathan Kuek graduated in 2018 with a Bachelor of Psychology (Hons) degree from James Cook University in Australia. He specializes in recovery approaches relevant to the management and understanding of mental health conditions. His undergraduate training was in the field of psychology. He currently is a doctoral candidate and mental health researcher at the University of Sydney, and a research assistant at the Institute of Mental Health Singapore at the Department of Clinical Governance and Quality since 2019. His main research interests include personal recovery, peer support, ethics and mental health stigma. His current doctoral research revolves around establishing a preliminary understanding of mental health personal recovery in Singapore. In his free time, he serves as the research chair for the Singapore Psychological Society, working to elevate the standing of psychological research in Singapore. 